\title{
OPENCHEM: A DEEP LEARNING TOOLKIT FOR COMPUTATIONAL CHEMISTRY AND DRUG DESIGN
}

\author{
Mariya Popova, ${ }^{1,2^{*}}$ Boris Ginsburg, ${ }^{3}$ Alexander Tropsha,$^{4}$ and Olexandr Isayev ${ }^{1,2, *}$ \\ ${ }^{1}$ Computational Biology Department, School of Computer Science, Carnegie Mellon University, Pittsburgh, \\ Pennsylvania, 15213 \\ ${ }^{2}$ Department of Chemistry, Mellon College of Science, Carnegie Mellon University, Pittsburgh, Pennsylvania, 15213 \\ ${ }^{3}$ NVIDIA Corporation, Santa Clara, California, 95050 \\ ${ }^{4}$ UNC Eshelman School of Pharmacy, University of North Carolina at Chapel Hill, Chapel Hill, North Carolina, 27599 \\ *Correspondence: mpopova@andrew.cmu.edu (M.P); olexandr@olexandrisayev.com (O.I.)
}

\section{Supporting Information}

Table S1. Description of standard training parameters for all models that are not related to the model architecture.

VARIABLE

TASK

TASK
TRAIN_DATA_LAYER

VAL_DATA_LAYER

PRINT_EVERY

SAVE_EVERY

LOGDIR

USE_CLIP_GRAD

MAX_GRAD_NORM

BATCH_SIZE

NUM_EPOCHS
DATA TYPE string

Specifies the task to be solved by the model

PyTorch dataset Contains training data

PyTorch dataset Contains validation data

int

int

string

boolean

float

int

int

used

Specifies the size of batches for training
DESCRIPTION

COMMENT
Could be classification, regression, multitask or generation

Could be None in evaluation mode

Could be None in training mode

By default, logs are printed every epoch

By default model is saved every 5 epochs

In evaluation mode, the model will be loaded from the latest checkpoint in this directory

By default, gradient clipping is not applied

N.A. if gradient clipping is not applied

Specifies the number of epochs for training.
Required parameter, no default value

Could be None in evaluation mode 
EVAL_METRICS

OPTIMIZER
Python function

PyTorch optimizer
A user-defined function that will

be used to calculate evaluation metrics.

Specifies an optimizer that will be used for training the model
Could be None in the training model. Majority of evaluation metrics can be found in Python scikit-learn package CITE Could be None in evaluation mode

\section{GraphCNN model description}

Each molecular graph is represented by two matrices - adjacency matrix $A$ and node attribute matrix $H$. Adjacency matrix $A$ is a binary matrix of size $N \times N$, where $N$ is the number of atoms in a molecule. Element $A_{i j}$ of adjacency matrix encodes if there is an edge between atom $i$ and atom $j$, i.e.:

$$
A_{i j}=\left\{\begin{array}{c}
1, \text { if there is an edge between } i \text { and } j, \\
0, \text { otherwise. }
\end{array}\right.
$$

The second matrix is a node attributes matrix $H$, which has size $N \times D$, where $D$ is the number of attributes calculated for every atom. The attributes encode physical and chemical properties of atoms in the context of the whole molecule.

Graph convolutions aggregate information from the node attribute matrix considering the nodes adjacency, i.e., only features from the neighboring atoms are convolved. A single layer of graph convolutions has the following form:

$$
H^{\prime}=\sigma(A H W)
$$

where $H^{\prime}$ is the new node attribute matrix of size $N \times D^{\prime}, W$ is the matrix of parameters of the convolution of size $D \times D^{\prime}$ and $\sigma$ is a nonlinearity function such as tanh.

A single graph convolution operation transforms the initial node attribute matrix $H$ of size $N \times D$ into a new matrix $H^{\prime}$ of size $N \times D^{\prime}$. In the initial matrix $H$ row number $i$ contains the information only about atom number $i$, while each row in the new matrix $H^{\prime}$ includes information on the corresponding atom and its neighbors.

Applying another graph convolution operation on matrix $H^{\prime}$ can be applied further transforms it into the new node attributes' matrix $H^{\prime \prime}$ and increase the receptive field by aggregating information from every node, its neighbors, and also the neighbors of the neighbors. Thus, the sequential application of graph convolution operations results in aggregation of the graph-level information in every row of the final node attribute matrix.

The final node attribute matrix is then converted into an embedding vector for the molecular graph using a pooling operation, such as averaging across all rows of matrix $H$ :

$$
h=\frac{1}{N} \sum_{i=1}^{N} H_{i} .
$$

